\title{
Chemical spots in the absence of magnetic field in the binary HgMn star 66 Eridani`
}

\author{
V. Makaganiuk ${ }^{1}$, O. Kochukhov ${ }^{1}$, N. Piskunov ${ }^{1}$, S. V. Jeffers ${ }^{2}$, C. M. Johns-Krull ${ }^{3}$, C. U. Keller ${ }^{2}$, M. Rodenhuis ${ }^{2}$, \\ F. Snik ${ }^{2}$, H. C. Stempels ${ }^{1}$, and J. A. Valenti ${ }^{4}$
}

\author{
1 Department Physics and Astronomy, Uppsala University, Box 516, 75120 Uppsala, Sweden \\ e-mail: vitaly.makaganiuk@fysast.uu.se \\ 2 Sterrekundig Instituut, Universiteit Utrecht, PO Box 80000, 3508 TA Utrecht, The Netherlands \\ 3 Department of Physics and Astronomy, Rice University, 6100 Main Street, Houston, TX 77005, USA \\ ${ }^{4}$ Space Telescope Science Institute, 3700 San Martin Dr, Baltimore MD 21211, USA
}

Received 13 December 2010 / Accepted 16 February 2011

\begin{abstract}
Context. According to our current understanding, a subclass of the upper main-sequence chemically peculiar stars, called mercurymanganese $(\mathrm{HgMn})$, is non-magnetic. Nevertheless, chemical inhomogeneities were recently discovered on their surfaces. At the same time, no global magnetic fields stronger than $1-100 \mathrm{G}$ are detected by systematic studies.

Aims. The goals of our study are to search for a magnetic field in the HgMn binary system 66 Eri and to investigate chemical spots on the stellar surfaces of both components.

Methods. Our analysis is based on high-quality spectropolarimetric time-series observations obtained during 10 consecutive nights with the HARPSpol instrument at the ESO 3.6-m telescope. To increase the sensitivity of the magnetic field search we employed a least-squares deconvolution (LSD). We used spectral disentangling to measure radial velocities and study the line profile variability. Chemical spot geometry was reconstructed using multi-line Doppler imaging.

Results. We report a non-detection of magnetic field in 66 Eri, with error bars 10-24 G for the longitudinal field. Circular polarization profiles also do not indicate any signatures of complex surface magnetic fields. For a simple dipolar field configuration we estimated an upper limit of the polar field strength to be 60-70 G. For the HgMn component we found variability in spectral lines of Ti, Ba, $\mathrm{Y}$, and $\mathrm{Sr}$ with the rotational period equal to the orbital one. The surface maps of these elements reconstructed with the Doppler imaging technique show a relative underabundance on the hemisphere facing the secondary component. The contrast of chemical inhomogeneities ranges from 0.4 for Ti to 0.8 for $\mathrm{Ba}$.
\end{abstract}

Key words. stars: chemically peculiar - stars: individual: HD 32964 - stars: variables: general - binaries: spectroscopic

\section{Introduction}

The phenomenon of spot formation on the stellar surfaces is known for a long time. Detailed studies of stars that exhibit surface features showed that the formation of temperature or chemical spots is closely related to the presence of magnetic field in the stellar atmospheres (Donati \& Landstreet 2009). Nevertheless, there are exceptional cases when spectropolarimetric studies of high sensitivity detect no magnetic field, but spots are still present on the stellar surface. This is observed in chemically peculiar (CP) stars of mercury-manganese $(\mathrm{HgMn})$ type.

The first discovery of spots in a HgMn star was reported for $\alpha$ And (Adelman et al. 2002), which also shows an evolution of spot structure (Kochukhov et al. 2007). Currently we know six other spotted HgMn stars: HR 1185 and HR 8723 (Kochukhov et al. 2005), AR Aur (Hubrig et al. 2006; Folsom et al. 2010), HD 11753, HD 53244 and HD 221507 (Briquet et al. 2010). All these stars exhibit spots of mostly heavy chemical elements. According to theoretical models adopted for CP stars, physical processes responsible for the spot formation are associated with a magnetic field. To enlarge the sample of spotted $\mathrm{HgMn}$ stars

* Based on observations collected at the European Southern Observatory, Chile (ESO program 084.D-0338). studied in detail, we have performed a comprehensive analysis of another HgMn star.

66 Eri (HR 1657, HD 32964, HIP 23794) is a well-known binary system with nearly identical components. Using the radial velocities determined by Frost \& Struve (1924) and his own observations, Young (1976) found the orbital period of the system to be $P \approx 5.523$ days, which was improved by the later studies (Yushchenko et al. 2001; Catanzaro \& Leto 2004). The orbital analysis yields a mass ratio $M_{\mathrm{B}} / M_{\mathrm{A}}^{1}$ very close to unity (Yushchenko et al. 2001; Catanzaro \& Leto 2004).

Photometric studies of 66 Eri are controversial. Young (1976) has estimated the times of possible eclipses based on the orbital elements of the system. His photometric observations at expected times of eclipses showed no variability. Young concluded that the orbital inclination angle is less than $80^{\circ}$. Later, Schneider (1987) detected weak variability with a period different from the orbital one. This weak photometric variability was not confirmed by Yushchenko et al. (1999), who in turn suggested variability with half of the orbital period.

Berghofer \& Schmitt (1994) reported this star as an X-ray source. In the later study Hubrig et al. (2001) detected the third,

\footnotetext{
1 Throughout our study we denote the more massive component (HgMn star, the primary) with the letter "A". The less massive secondary component is denoted by " $\mathrm{B}$ ".
} 
low-mass companion, which is likely to be the actual source of the X-ray radiation coming from the system. This was directly confirmed with the Chandra X-ray imaging by Stelzer et al. (2006).

The early chemical analysis of 66 Eri by Young (1976) showed overabundances of $\mathrm{Hg}, \mathrm{Cr}$ and $\mathrm{Y}$ in the primary component, while the secondary was reported to be normal. Woolf \& Lambert (1999) determined Hg abundance in a large number of HgMn stars, including 66 Eri. They confirmed the overabundance of mercury in the primary component. A detailed abundance analysis of 66 Eri was presented by Yushchenko et al. (1999), who studied chemical composition of both components of the binary system. Combining spectroscopy and photometry, they determined $T_{\text {eff }}=11100 \pm 100 \mathrm{~K}$ and $T_{\text {eff }}=10900 \pm 100 \mathrm{~K}$ for the component B and A, respectively. The similarity of the physical parameters of the components also follows from their luminosity ratio $L_{\mathrm{B}} / L_{\mathrm{A}}=0.95 \pm 0.05$. The rotational velocity of both stars is equal to $v_{\mathrm{e}} \sin i=17 \mathrm{~km} \mathrm{~s}^{-1}$ (Yushchenko et al. 1999).

66 Eri is very similar to another double-line binary with an HgMn component - AR Aur (Khokhlova et al. 1995). Despite the fact that no magnetic field was found in AR Aur, it shows chemical inhomogeneities (Folsom et al. 2010). The similarity in the two HgMn SB2 stars inspired us to analyse 66 Eri with new high-precision observational data.

The paper is structured as follows. In Sect. 2 we describe the observational material and the data reduction process. Section 3 presents the least-squares deconvolution analysis. In Sect. 4 we discuss the measurements of the magnetic field in 66 Eri. Section 5 describes the spectral disentangling. A determination of the system parameters is given in Sect. 6. Section 7 presents an analysis of the spectral variability. An interpretation of the line variability in terms of surface chemical maps is presented in Sect. 8. The paper ends with a summary of the main results and discussion in Sect. 9.

\section{Observations and data reduction}

Using the newly built polarimeter HARPSpol (Snik et al. 2008, 2010) attached to the HARPS spectrometer (Mayor et al. 2003) at the ESO 3.6-m telescope we obtained high-quality observations at ten phases, covering the full orbital period of 66 Eri. The resolution of spectra is $R=115000$ and a typical $S / N$ is 200-300 at $\lambda \approx 5200 \AA$.

The HARPS detector is a mosaic of two $2 \mathrm{~K} \times 4 \mathrm{~K}$ CCDs, allowing us to record 45 and 26 polarimetric echelle orders on the blue and red CCD, respectively. The calibration set included 20 bias exposures, 20 flat fields and two ThAr frames for each night. The flat fields and comparison spectra were recorded using the circular polarization mode.

All science frames of 66 Eri were obtained in circular polarization, covering the wavelength range of 3780-6913 $\AA$ with a small gap at 5259-5337 $\AA$. Each observation of the star was split into four sub-exposures corresponding to the $45^{\circ}, 135^{\circ}, 225^{\circ}$ or $315^{\circ}$ position of the quarter-wave plate relative to the beamsplitter. The length of individual sub-exposures varied between 200 and $300 \mathrm{~s}$, depending on the weather conditions.

The ESO reduction pipeline was not available at that time, so we employed REDUCE package (Piskunov \& Valenti 2002), written in IDL, to perform a number of standard steps to reduce and calibrate our cross-dispersed echelle spectra. We averaged and subtracted bias images from the master flat and science frames. To locate spectral orders, REDUCE uses a cluster analysis method in conjunction with the master flat image. For the correction of pixel-to-pixel sensitivity variations in the science images the code normalizes the master flat field. Finally, REDUCE removes the scattered light and performs the optimal extraction of the stellar spectra as described by Piskunov \& Valenti (2002).

The HARPS spectrometer is well-known for its stability, which is of the order of $1 \mathrm{~m} \mathrm{~s}^{-1}$. Taking this into account, it is sufficient for our study to use only one ThAr spectrum obtained during each night to do the wavelength calibration. Employing 700-900 ThAr lines we constructed a 2D wavelength solution with an internal wavelength calibration accuracy of $18-21 \mathrm{~m} \mathrm{~s}^{-1}$. The final step before the Stokes parameters derivation is continuum normalization. The continuum level is set by dividing each spectrum by a smooth, slowly varying function, obtained by fitting the upper envelope of the blaze-shape-corrected, spliced spectrum.

To derive Stokes $V$ parameter we used the ratio method described by Bagnulo et al. (2009). This method minimizes spurious polarization effects by an appropriate combination of the two physical beams recorded for four different retarder quarterwave plate positions. Along with the polarization we also derive a diagnostic null spectrum. It is obtained by combining the polarization signal in individual sub-exposures destructively, thus showing the residual instrumental polarization remaining after application of the ratio method. We used the null spectrum in the same analysis steps as the Stokes $V$ spectrum, thus providing a realistic estimate of possible errors.

The combination of eight spectra in four individual subexposures provided a convenient possibility to detect and remove remaining cosmic hits that otherwise seriously distort the final Stokes spectra. Affected pixels were identified by their high deviation from the median and were substituted by the latter. Since our exposure times were relatively short, only 1-2 pixels required correction in each echelle order.

\section{Least-squares deconvolution}

Previous attempts to find magnetic fields in $\mathrm{HgMn}$ stars set an upper limit on the longitudinal magnetic field below $100 \mathrm{G}$ (Shorlin et al. 2002; Wade et al. 2006; Aurière et al. 2010; Folsom et al. 2010; Makaganiuk et al. 2011). The fields of this strength are undetectable in individual spectral lines. In this case the sensitivity of magnetic field search can be increased by adding information from many spectral lines. Such a multiline method has been implemented by Donati et al. (1997) in the so-called least-squares deconvolution (LSD). This technique assumes an identical shape of spectral lines and represents observations as a superposition of profiles scaled by appropriate weight. With this method it becomes possible to derive a highprecision average profile, increasing the signal-to-noise ratio by a factor of up to 1000 . The latest studies of weak magnetic fields proved the LSD technique to be very effective in detecting fields weaker than $1 \mathrm{G}$ (Aurière et al. 2009; Lignières et al. 2009).

To construct a line mask required by LSD, we extracted the line list from VALD (Piskunov et al. 1995) using an atmospheric model with the effective temperature of $10900 \mathrm{~K}$ and abundance of chemical elements according to the results of Yushchenko et al. (1999) for the more massive star. We note that LSD analysis is very weakly sensitive to the choice of $T_{\text {eff }}$ and abundances adopted for the mask.

A line mask is a set of the laboratory line wavelengths and corresponding weights. For the Stokes I spectra, the weight is a residual depth of an individual line, given by VALD. For the Stokes $V$ spectra the weight is the product of central depth, the 

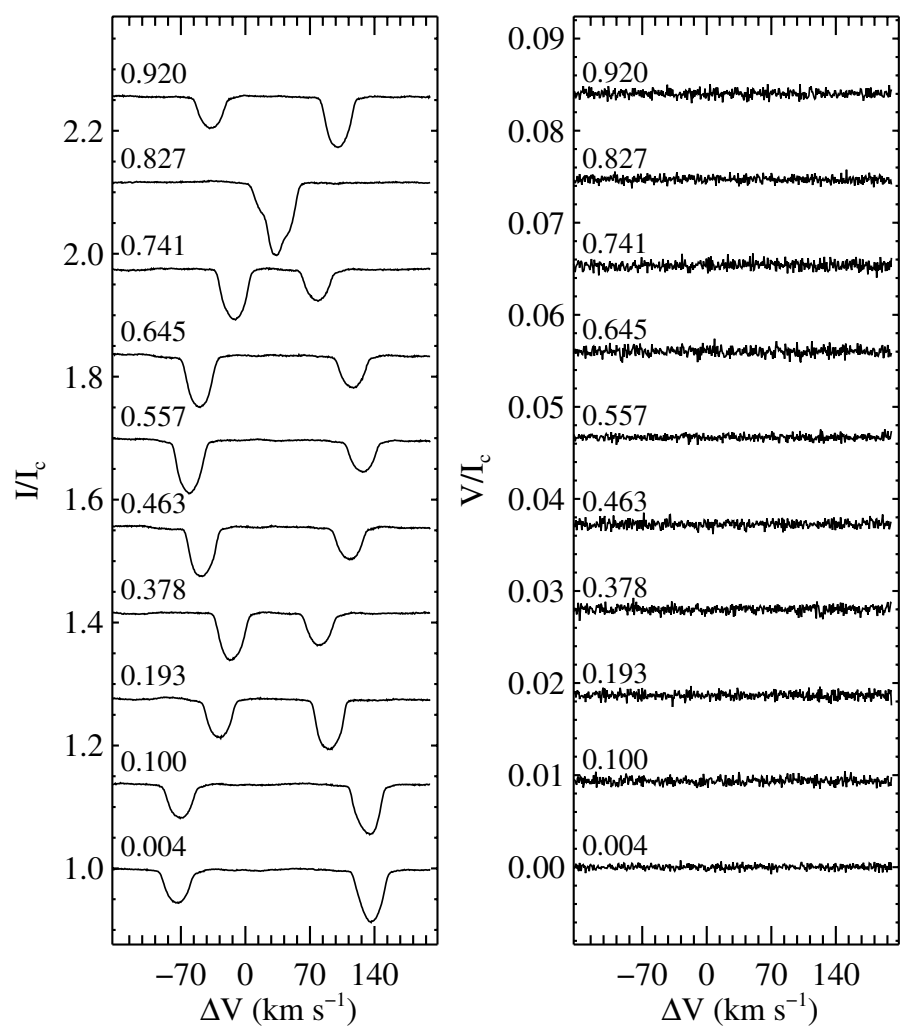

Fig. 1. Variation of the LSD Stokes I profiles (left panel) and LSD Stokes $V$ (right panel) with the orbital/rotational phase. For displaying purposes we shifted the Stokes $I$ and $V$ profiles vertically and multiplied the latter by a factor of 15 . Phases are given on the left, above each profile.

line wavelength normalized by $\lambda=4800 \AA$, and an effective Landé factor of a given spectral line. Spectral lines with the central depth lower than a given cutoff factor are excluded from the line mask. By setting the cutoff criterion to 0.1 , we produced a line mask containing 717 spectral lines.

We used the LSD code by Kochukhov et al. (2010) to compute LSD Stokes $I, V$, and null profiles. Profiles were computed in the velocity range from -170 to $200 \mathrm{~km} \mathrm{~s}^{-1}$ with a step of $0.8 \mathrm{~km} \mathrm{~s}^{-1}$. This value corresponds to the average pixel scale of HARPSpol spectra. The errors of LSD profiles were calculated by propagating the uncertainties of individual pixels obtained at the reduction stage.

In general there is a mismatch between a model LSD spectrum and real observations owing to coarse assumptions of the LSD technique. To account for these systematic errors we scaled the uncertainties of the calculated LSD profiles by the reduced $\chi^{2}$ as described by Wade et al. (2000). The re-scaled LSD profile uncertainties are used for the estimation of the longitudinal magnetic field measurements.

In Fig. 1 we present the LSD Stokes $I$ and $V$ obtained for ten orbital phases of 66 Eri.

\section{Magnetic field measurements}

The longitudinal magnetic field was determined from the first moment of the LSD Stokes $V$ profile (Kochukhov et al. 2010). Because of the contribution of blends the continuum level of the raw LSD Stokes I profiles is slightly offset from unity. We used a constant factor to correct the continuum level. This factor is applied to the LSD $V$ and null profiles. This correction

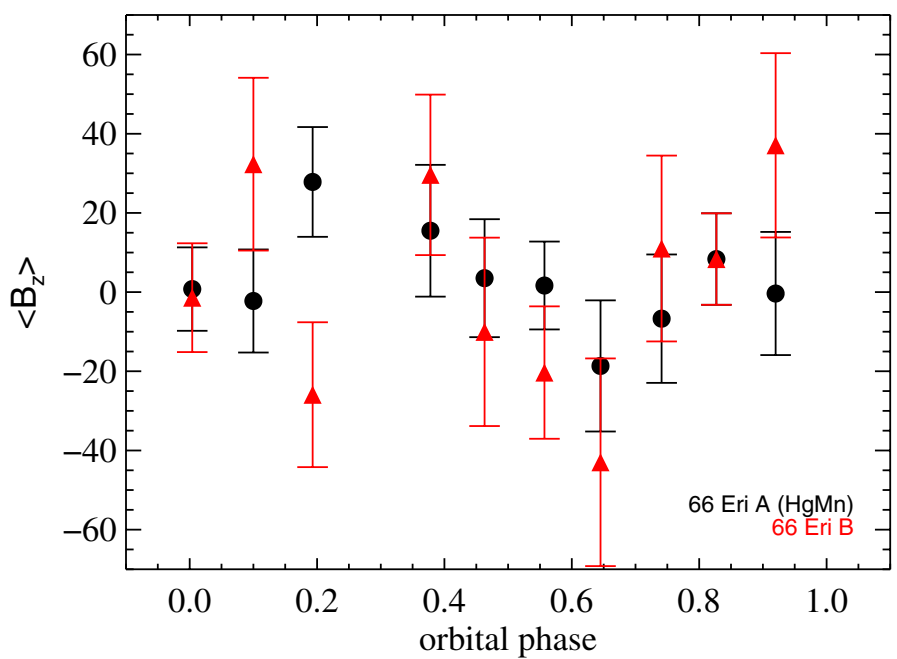

Fig. 2. Measurements of the longitudinal magnetic field with the corresponding error bars. Circles show the $\left\langle B_{z}\right\rangle$ values for the primary component ( $\mathrm{HgMn}$ star) and triangles represent the measurements for the secondary.

changes the value of the longitudinal magnetic field and its error by $2-3 \mathrm{G}$.

For nine LSD $V$ profiles we could measure the longitudinal field for each component separately. At the phase 0.827 (see Fig. 1) we measured both components as a single star. The results of the longitudinal magnetic field measurements are presented in Table 1. Its columns contain the following information: the heliocentric Julian date of observation, the signal-tonoise ratio of the original spectra measured around $5200 \AA$, the signal-to-noise ratio of the LSD $V$ profiles, an orbital phase, determined from the binary solution discussed below. The next three columns represent measurements for the $\mathrm{HgMn}$ star (component A) as follows: the mean longitudinal magnetic field inferred from the LSD Stokes $V$, the longitudinal field inferred from the null profile, and a false alarm probability (FAP, see below). The last three columns give the same information for the component B.

The errors of the magnetic field measurements were obtained by the standard error propagation, using the uncertainties of the LSD $V$ profiles. The mean error of the longitudinal field measurements is $\sim 14 \mathrm{G}$ for the HgMn component and $\sim 20 \mathrm{G}$ for the secondary. For the $\mathrm{HgMn}$ star the longitudinal field value reaches the confidence level of $2 \sigma$ only at the orbital phase 0.193 . For the secondary star the measurements of the magnetic field do not exceed $\sim 1.5 \sigma$ level for most of the orbital phases. Thus, none of the individual $\left\langle B_{z}\right\rangle$ measurements indicates the presence of a magnetic field in 66 Eri.

Our magnetic field measurements for both components with their respective error bars are presented in Fig. 2 as a function of orbital phase. The actual values of the longitudinal field measurements do not exceed the level of $3 \sigma$, so the periodic-like fluctuation of magnetic field measurements in both components are fully attributed to errors.

Assuming a dipolar configuration of the magnetic field, we made an assessment of the upper limit of its strength, which would be consistent with the entire set of $\left\langle B_{z}\right\rangle$ measurements. Using a relation between the longitudinal field and parameters of an oblique dipole (Leroy et al. 1994), we estimated the strength of dipolar magnetic field to be 60-70 $\mathrm{G}$ with an uncertainty of $50-70 \mathrm{G}$ for both stars. However, based on the reduced $\chi^{2}$ statistics, the dipolar fit has no advantage over the null hypothesis. 
Table 1. Magnetic field analysis of 66 Eri.

\begin{tabular}{rccc|rrr|rrr}
\hline \hline & & & \multicolumn{3}{c}{$\mathrm{A}$} \\
$\mathrm{HJD}-24 \times 10^{5}$ & $S / N$ & $S / N(\mathrm{LSD})$ & Phase & $\left\langle B_{z}\right\rangle(\mathrm{V}), \mathrm{G}$ & $\left\langle B_{z}\right\rangle(\mathrm{null}), \mathrm{G}$ & $\mathrm{FAP} \times 10$ & $\left\langle B_{z}\right\rangle(\mathrm{V}), \mathrm{G}$ & $\left\langle B_{z}\right\rangle(\mathrm{null}), \mathrm{G}$ & $\mathrm{FAP} \times 10$ \\
\hline 55202.725056 & 245 & 2638 & 0.193 & $28 \pm 14$ & $7 \pm 14$ & 4.92 & $-26 \pm 18$ & $39 \pm 18$ & 5.49 \\
55203.744467 & 299 & 2674 & 0.378 & $30 \pm 20$ & $-22 \pm 20$ & 9.29 & $16 \pm 17$ & $8 \pm 17$ & 7.73 \\
55204.737221 & 320 & 3304 & 0.557 & $-20 \pm 17$ & $32 \pm 17$ & 0.61 & $2 \pm 11$ & $-12 \pm 11$ & 9.63 \\
55205.755838 & 185 & 2409 & 0.741 & $11 \pm 23$ & $61 \pm 23$ & 3.03 & $-7 \pm 16$ & $9 \pm 16$ & 2.60 \\
55206.736354 & 304 & 2671 & 0.920 & $0 \pm 16$ & $15 \pm 16$ & 2.59 & $37 \pm 23$ & $27 \pm 23$ & 3.21 \\
55207.735135 & 315 & 2929 & 0.100 & $-2 \pm 13$ & $-25 \pm 13$ & 0.80 & $32 \pm 22$ & $-16 \pm 22$ & 3.24 \\
55209.741479 & 261 & 2519 & 0.463 & $-10 \pm 24$ & $3 \pm 24$ & 9.75 & $4 \pm 15$ & $-13 \pm 15$ & 1.76 \\
55210.746427 & 216 & 2225 & 0.645 & $-43 \pm 26$ & $0 \pm 26$ & 2.11 & $-19 \pm 17$ & $5 \pm 17$ & 9.74 \\
55211.750571 & 241 & 2965 & 0.827 & $8 \pm 12$ & $0 \pm 12$ & 3.62 & $8 \pm 12$ & $0 \pm 12$ & 3.62 \\
55212.727307 & 380 & 3901 & 0.004 & $-1 \pm 14$ & $40 \pm 14$ & 8.28 & $1 \pm 11$ & $-14 \pm 11$ & 6.58 \\
\hline
\end{tabular}

At the same time, much more complex magnetic fields can exist in the atmospheres of both stars. To investigate the possible presence of these complex magnetic structures, which might yield negligible $\left\langle B_{z}\right\rangle$, we employed the FAP analysis of LSD profiles. FAP is a $\chi^{2}$ statistical estimate, which assesses if the observed structure in the Stokes $V$ line profile is produced by a random noise. Following the definition by Donati et al. (1997), these numbers should be interpreted as follows. No detection if FAP $>10^{-3}$, marginal detection if $10^{-3} \leq$ FAP $\leq 10^{-5}$ and definite detection if FAP $<10^{-5}$. The smallest FAP obtained for $\mathrm{HgMn}$ star is 0.08 . Thus, none of the measurements indicates the presence of a magnetic signal. A visual examination of each LSD Stokes $V$ profile also does not suggest a magnetic field detection.

To ensure that the LSD circular polarization profiles were not affected by a spurious polarization, we analysed LSD null spectra at each phase. The $\left\langle B_{z}\right\rangle$ and FAP measurements were done in the same way as for LSD Stokes $V$ profiles. The $\left\langle B_{z}\right\rangle$ errors for the null spectra are fully consistent with those inferred from the circular polarization profiles. The absence of a signal in the null spectrum means that our scientific data are not visibly affected by significant spurious polarization.

\section{Spectral disentangling}

Because of the orbital motion, the relative shift of the spectral lines of the components in the composite spectrum varies from zero to $\sim 200 \mathrm{~km} \mathrm{~s}^{-1}$, which can be seen from the LSD I profiles in Fig. 1. In addition to this, the primary component shows an intrinsic line profile variability. Since one of the main aims of this study is to investigate line profile variability in 66 Eri, it is important to separate the effect of the spectral line blending caused by the orbital motion from an intrinsic variability.

To separate different variability effects we employ the procedure of spectral disentangling described by Folsom et al. (2010). It combines information from all orbital phases and yields radial velocities (RV) for both components, their average separated spectra and standard deviation in the reference frame of each component. Figure 3 shows an example of spectral disentangling in the region 4355-4380 A containing several variable lines.

The spectral disentangling procedure provided a new set of high-precision RV measurements for both components of 66 Eri. These data, presented in Table 2, have typical error bars of $0.1-0.2 \mathrm{~km} \mathrm{~s}^{-1}$. We combined our RVs with the velocities published by Young (1976), Yushchenko et al. (1999, 2001), and Catanzaro \& Leto (2004), deriving improved orbital parameters of the system. The new orbital elements are listed in Table 3, while Fig. 4 compares the respective predicted RV curves with
Table 2. Radial velocities of the primary and secondary components of 66 Eri.

\begin{tabular}{lrrr}
\hline \hline & \multicolumn{1}{c}{$\mathrm{A}$} & \multicolumn{1}{c}{$\mathrm{B}$} \\
$\mathrm{HJD}-24 \times 10^{5}$ & Phase & $V_{\mathrm{r}}, \mathrm{km} \mathrm{s}^{-1}$ & $V_{\mathrm{r}}, \mathrm{km} \mathrm{s}^{-1}$ \\
\hline 55202.725056 & 0.193 & $91.26 \pm 0.19$ & $-27.92 \pm 0.18$ \\
55203.744467 & 0.378 & $-14.91 \pm 0.20$ & $80.34 \pm 0.14$ \\
55204.737221 & 0.557 & $-60.30 \pm 0.14$ & $127.11 \pm 0.17$ \\
55205.755838 & 0.741 & $-12.25 \pm 0.13$ & $77.98 \pm 0.16$ \\
55206.736354 & 0.920 & $100.36 \pm 0.11$ & $-37.29 \pm 0.11$ \\
55207.735135 & 0.100 & $132.44 \pm 0.15$ & $-70.55 \pm 0.13$ \\
55209.741479 & 0.463 & $-46.91 \pm 0.21$ & $113.26 \pm 0.25$ \\
55210.746427 & 0.645 & $-49.79 \pm 0.09$ & $116.64 \pm 0.16$ \\
55211.750571 & 0.827 & $40.04 \pm 0.09$ & $24.58 \pm 0.21$ \\
55212.727307 & 0.004 & $-74.02 \pm 0.08$ & $135.94 \pm 0.08$ \\
\hline
\end{tabular}

Table 3. Results of the spectroscopic orbital analysis of 66 Eri.

\begin{tabular}{lc}
\hline \hline Parameter & Value \\
\hline$P_{\text {orb }}(\mathrm{d})$ & $5.5226013 \pm 0.0000020$ \\
$T_{0}(\mathrm{~d})$ & $41356.499 \pm 0.017$ \\
$K_{\mathrm{A}}\left(\mathrm{km} \mathrm{s}^{-1}\right)$ & $102.83 \pm 0.20$ \\
$K_{\mathrm{B}}\left(\mathrm{km} \mathrm{s}^{-1}\right)$ & $100.35 \pm 0.19$ \\
$\gamma\left(\mathrm{km} \mathrm{s}^{-1}\right)$ & $32.28 \pm 0.10$ \\
$e$ & $0.0844 \pm 0.0013$ \\
$\omega\left({ }^{\circ}\right)$ & $160.9 \pm 1.1$ \\
$M_{\mathrm{B}} / M_{\mathrm{A}}$ & $0.976 \pm 0.003$ \\
$M_{\mathrm{B}} / M_{\odot} \sin ^{3} i_{\text {orb }}$ & $2.345 \pm 0.008$ \\
$M_{\mathrm{A}} / M_{\odot} \sin ^{3} i_{\text {orb }}$ & $2.403 \pm 0.008$ \\
$a_{\mathrm{B}} / R_{\odot} \sin i_{\text {orb }}$ & $11.185 \pm 0.022$ \\
$a_{\mathrm{A}} / R_{\odot} \sin i_{\text {orb }}$ & $10.916 \pm 0.020$ \\
\hline
\end{tabular}

the actual measurements. The standard deviation of the orbital fit is $2.5 \mathrm{~km} \mathrm{~s}^{-1}$ for the primary and $2.8 \mathrm{~km} \mathrm{~s}^{-1}$ for the secondary. Compared to the previous orbital analysis by Catanzaro \& Leto (2004), we significantly improved the accuracy of the orbital period and of the velocity amplitudes, obtaining a mass ratio with a precision better than $1 \%$.

\section{Fundamental parameters of the components}

The very similar properties of the 66 Eri components make it challenging to determine their parameters individually. The previous detailed study of the system by Yushchenko et al. (1999) suggested that the slightly more massive component, the $\mathrm{HgMn}$ star, is also more luminous, but cooler by $200 \pm 140 \mathrm{~K}$ than the other star. The sign of this spectroscopically determined temperature difference is impossible to reconcile with the main sequence status of co-evolving binary components.

We redetermined the fundamental parameters of 66 Eri using the Padova evolutionary tracks (Girardi et al. 2000) and taking 
V. Makaganiuk et al.: Magnetic fields and variability of 66 Eri

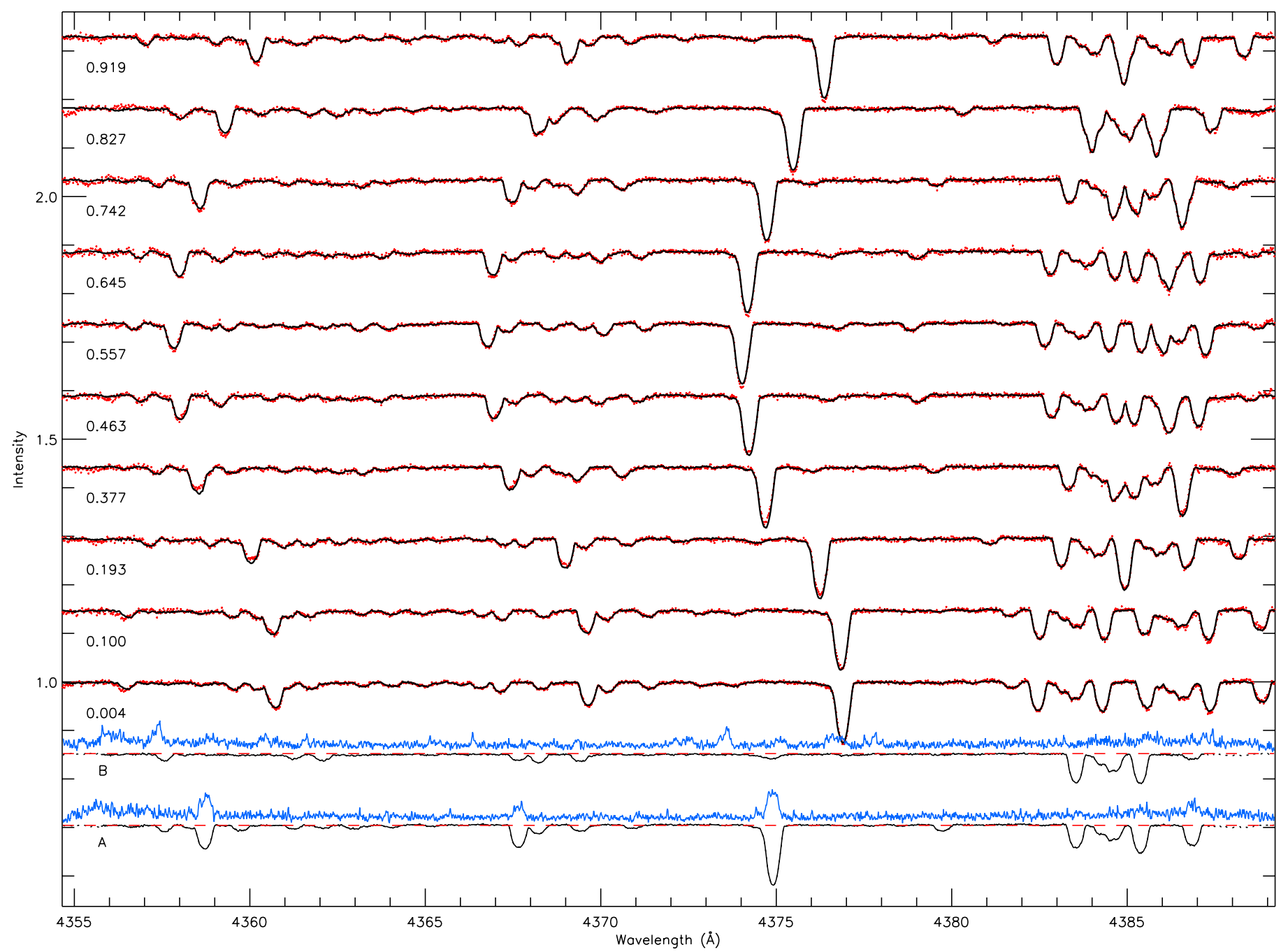

Fig. 3. Spectral disentangling procedure applied to 66 Eri. Dots show the observations and the solid line is the model spectrum for corresponding phase. Black solid lines at the bottom represent disentangled spectra for the primary and secondary components. The lines above the continuum level represent the standard deviations in the reference frames of components A and B. Peaks at the location of spectral lines in the standard deviation indicate the presence of intrinsic variability in a given line (Y II $4358.728 \AA$, Ti II $4367.652 \AA$, and Y $_{\text {II }} 4374.935 \AA$ in this spectral interval).

advantage of the improved orbital parameters determined in our study and an accurate Hipparcos parallax, $\pi=10.56 \pm 0.34$ mas, available for the system (van Leeuwen \& Fantino 2005). The primary component mass and the age of the system were found by interpolating in the set of evolutionary tracks for $Z=0.018$ to match the total Hipparcos luminosity, $L / L_{\odot}=98.2 \pm 6.3$, and the mean effective temperature, $\left\langle T_{\text {eff }}\right\rangle=11000 \mathrm{~K}$, determined by Yushchenko et al. (1999) from the photometric properties and spectral energy distribution of 66 Eri. The outcome of this analysis is illustrated in the upper panel of Fig. 5. Table 4 summarizes the resulting fundamental parameters of the components, with error bars reflecting the uncertainty of the total luminosity. Effective temperatures of the components are given without error bars because they are mainly sensitive to $\left\langle T_{\mathrm{eff}}\right\rangle$, for which no uncertainty was provided by Yushchenko et al. (1999).

Our analysis of the H-R diagram position of the components of 66 Eri suggests that the primary is more massive, hotter, and more luminous $\left(L_{\mathrm{A}} / L_{\mathrm{B}}=1.093\right)$, as one would expect for two co-evolving main sequence stars. Our temperature difference, $T_{\text {eff }}(\mathrm{A})-T_{\text {eff }}(\mathrm{B})=163 \mathrm{~K}$, is opposite to what was suggested by Yushchenko et al. (1999). Nevertheless, the results of their abundance analysis of 66 Eri components are unlikely to change substantially due to $\sim 200 \mathrm{~K}$ change of $T_{\text {eff }}$.

The evolutionary masses and radii allow us to determine the orbital and rotational inclination angles, testing synchronization of the system. We found the projected rotational velocities, $v_{\mathrm{e}} \sin i_{\mathrm{rot}}=17.1 \pm 0.2$ and $16.9 \pm 0.2 \mathrm{~km} \mathrm{~s}^{-1}$ for the component $\mathrm{A}$ and $\mathrm{B}$, respectively, from fitting the profiles of $\sim 20$ unblended lines in the 4400-4600 $\AA$ wavelength region. These results excellently agree with $v_{\mathrm{e}} \sin i_{\text {rot }}=17 \mathrm{~km} \mathrm{~s}^{-1}$ given by Yushchenko et al. (1999) for both stars. Since the radii are known, the oblique rotator relation yields $i_{\text {rot }} \sim 74^{\circ}$ assuming $P_{\text {rot }}=P_{\text {orb }}=5.5226$ (see lower panel in Fig. 5). On the other hand, from the orbital solution and evolutionary masses $i_{\text {orb }}=76^{\circ}$. This convergence to nearly identical inclination angles derived using independent methods is a strong indication that the rotation of the 66 Eri components and their orbital motion are coaxial and synchronized.

\section{Line profile variability}

The line profile variability search was performed based on the disentangled spectra of both components. We carefully 


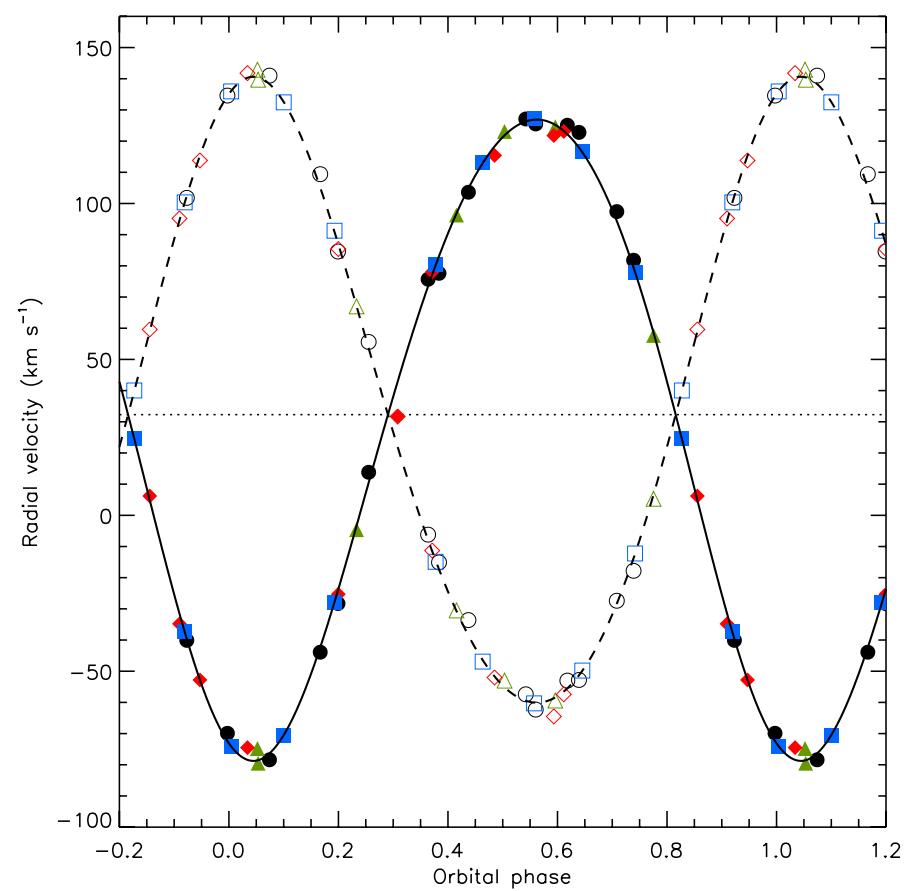

Fig. 4. Comparison of the radial velocities from different studies with our orbital solution. Filled symbols correspond to the secondary component and open ones to the primary. Circles represent measurements from Young (1976), triangles represent velocities from Yushchenko et al. $(1999,2001)$, diamonds are from Catanzaro \& Leto (2004), and squares represent our measurements. The lines show the theoretical radial velocity curve for the secondary (solid) and primary (dashed).

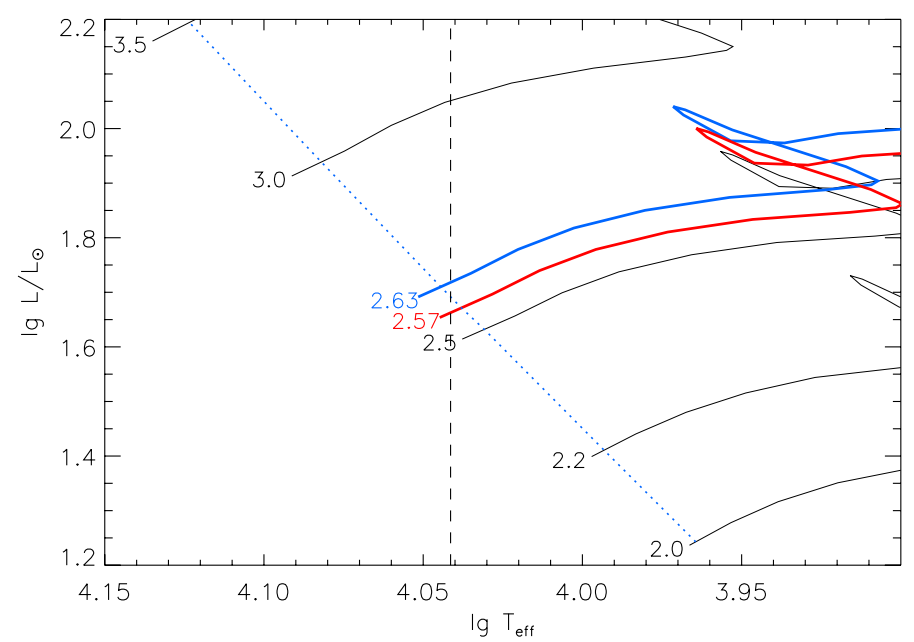

Fig. 5. Luminosity-effective temperature diagram constructed for the components of 66 Eri with the evolutionary calculations by Girardi et al. (2000). The thin solid curves represent the original evolutionary tracks, while thick curves show the tracks interpolated for the final component masses. The dotted line shows the isochrone for $\log t=7.47$ yr. The vertical dashed line corresponds to mean effective temperature of the components, $T_{\text {eff }}=11000 \mathrm{~K}$.

examined each spectral region, excluding hydrogen lines and the regions populated by the telluric lines. To diagnose the variability in spectral lines we computed standard deviation in the reference frame of each component. It is shown in Fig. 3 by a line above the average spectra of components A and B. An excess of standard deviation at the positions of spectral lines indicates their variability.
Table 4. Fundamental parameters of the primary and secondary components of 66 Eri.

\begin{tabular}{lcc}
\hline \hline Parameter & $\mathrm{A}$ & $\mathrm{B}$ \\
\hline $\log t(\mathrm{yr})$ & \multicolumn{2}{c}{$7.47 \pm 0.23$} \\
$M / M_{\odot}$ & $2.629 \pm 0.032$ & $2.566 \pm 0.032$ \\
$R / R_{\odot}$ & $1.948 \pm 0.063$ & $1.919 \pm 0.061$ \\
$L / L_{\odot}$ & $51.3 \pm 3.3$ & $46.9 \pm 3.0$ \\
$T_{\text {eff }}(\mathrm{K})$ & 11077 & 10914 \\
$\log g$ & $4.28 \pm 0.03$ & $4.28 \pm 0.03$ \\
$v_{\mathrm{e}} \sin i\left(\mathrm{~km} \mathrm{~s}^{-1}\right)$ & $17.1 \pm 0.2$ & $16.9 \pm 0.2$ \\
\hline
\end{tabular}

The variability search in spectral lines was performed for both components. We confirm that the secondary component does not exhibit any variability, while for the primary star we found variability in the spectral lines of Ti II, Sr II, Y II, and Ba II. This variability is generally weaker than found in other spotted HgMn stars. We suspect that a weak variability is also present in some $\mathrm{Cr}$ lines.

Earlier studies of HgMn stars (Adelman et al. 2002; Kochukhov et al. 2005) focused on the variability of $\mathrm{Hg}$ II line at $\lambda 3984 \AA$. Our analysis of this spectral line shows that, despite being fairly strong, it shows extremely weak variability in 66 Eri A.

The full list of variable lines is given in Table 5. The first column of this table gives a line identification, the second lists the corresponding laboratory wavelength. The last two columns provide the same information for occasional blending lines. Lines with marginal variability are marked with italics.

In Fig. 6 we show typical variable spectral lines of the four chemical elements with definite line profile changes. Spectra are plotted together with an average profile (dotted line). The strongest variability is found for $\mathrm{Sr}$ and $\mathrm{Ba}$ lines; the weakest is seen for Ti. The examination of the line profile variability showed that all spectral lines listed in Table 5 show the highest profile distortion relative to the mean spectrum at phases 0.193 and 0.378 . The variability of Ba II is also clearly seen for the phase 0.463 . In addition, the lines of $\mathrm{Y}_{\text {II }}$ and $\mathrm{Sr}$ II also marginally deviate from the corresponding average profiles at phases 0.827 , 0.920 and $0.741,0.827$ respectively.

The observed spectral variability occurs with the period of the orbital motion. For all four chemical elements with variable lines we found a distinct change of the radial velocity and, especially, an equivalent width with the orbital period. Since our observations cover only two rotation cycles, we cannot determine the variability period with a high precision. However, we observed that the integral properties of the variable lines and their shapes closely repeat after one orbital period. This strongly suggests that the orbital motion and the stellar rotation are synchronized.

\section{Doppler imaging}

To study the surface distribution of chemical elements in 66 Eri A, we applied the Doppler imaging (DI) method. We used the INVERS10 code (Piskunov \& Kochukhov 2002) to determine abundance maps, ignoring magnetic field. This analysis was applied to several non-blended spectral lines of Ti and Y that exhibit a significant variability and to all available spectral lines of $\mathrm{Sr}$ and $\mathrm{Ba}$. In total, we used two spectral lines of $\mathrm{Sr}$ II, two of Ba II, six lines of Y II, and nine spectral lines of Ti II. These lines are marked with bold font in Table 5. Multi-line DI is applied here to study spots in an HgMn star for the first time. This al- 
V. Makaganiuk et al.: Magnetic fields and variability of 66 Eri
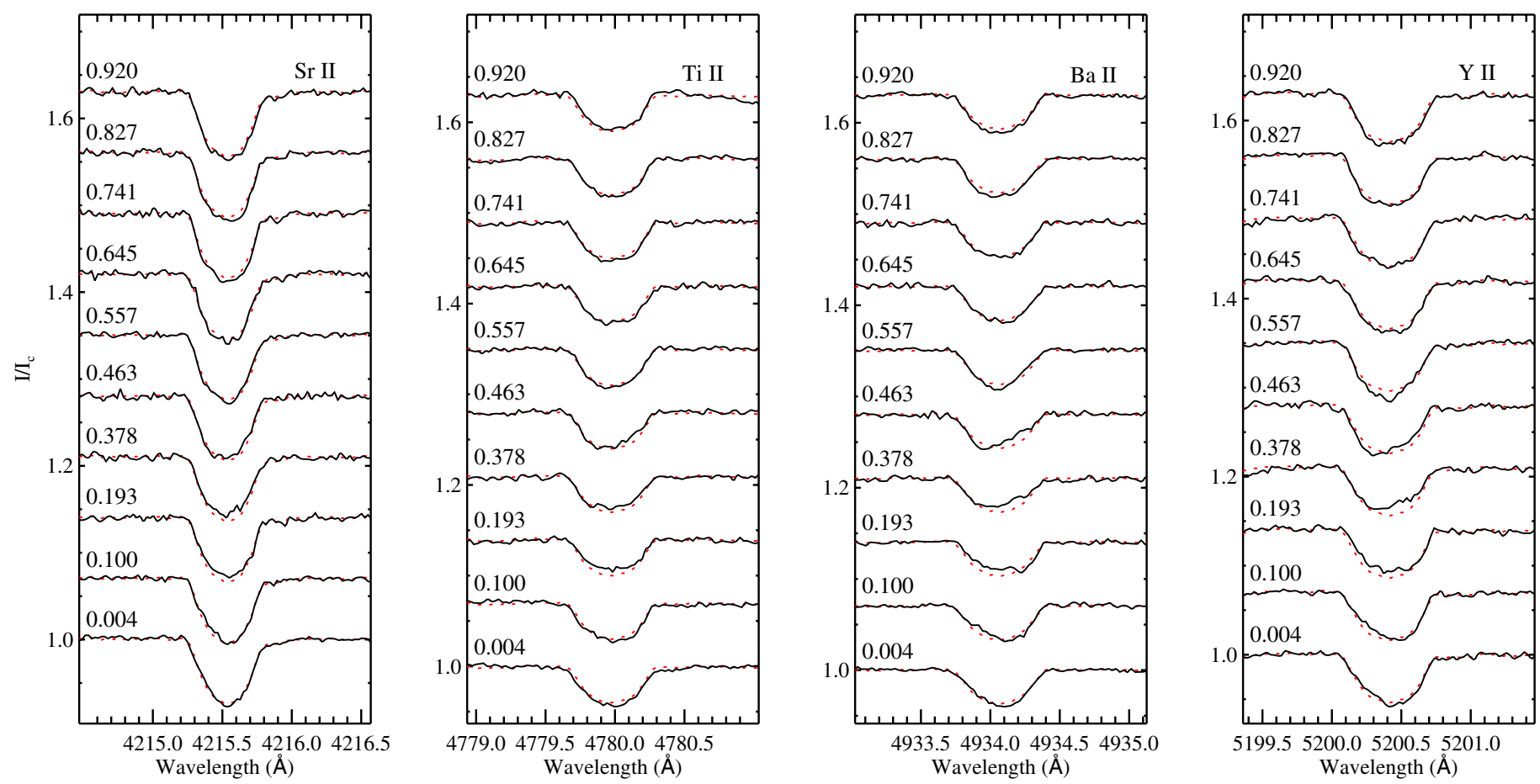

Fig. 6. Illustration of the line profile variability in the primary component (HgMn star) of 66 Eri. On the panels from left to right we show the

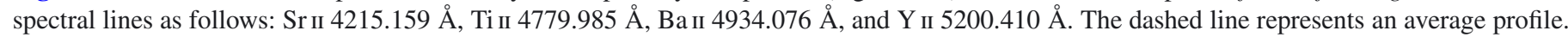
All profiles are shifted upwards for displaying purposes.

lowed us to reconstruct the surface maps with a higher precision than what was usually achieved using a single spectral line.

Doppler inversions were performed using the inclination angle of $75^{\circ}$, as determined earlier (Sect. 6). The initial abundances of $\mathrm{Ti}, \mathrm{Sr}, \mathrm{Y}$, and Ba were taken from Yushchenko et al. (1999). Spectra were calculated employing the revised model atmosphere of 66 Eri A with parameters $T_{\text {eff }}=11100 \mathrm{~K}$ and $\log g=4.25$. The rotational velocity was adjusted to give a better fit to the observed line profiles, yielding the final value of $v_{\mathrm{e}} \sin i=17.5 \pm 0.2 \mathrm{~km} \mathrm{~s}^{-1}$. The reconstructed surface maps are shown in Fig. 7. Figures 8-10 illustrate the comparison of observed and model line profiles.

First of all, we can see from these maps that there is a general asymmetry of the chemical composition for the two hemispheres of the star. For all four studied chemical elements we find a higher abundance at rotational phases from 0.5 to 0.1 and a lower abundance for phases $0.1-0.5$. Secondly, there is a different spot structure for the hemisphere with element overabundances.

The spots of $\mathrm{Ba}$ and other chemical elements except Ti extend from $-30^{\circ}$ to $60^{\circ}$ in latitude, showing the highest concentrations at the phase 0.65 . For $\mathrm{Y}$ and $\mathrm{Sr}$ we also find the secondary spot at the phases 0.95-1.05. The spots of $\mathrm{Sr}$ and $\mathrm{Ti}$ are more extended azimuthally, while the spots of $\mathrm{Ba}$ and $\mathrm{Y}$ are stretched meridionally. Sr is also the only element of the four that shows a relative overabundance at the pole. Finally, Ti shows the spot positions similar to $\mathrm{Sr}$, but with a discontinuity at the stellar equator even for the region of relative underabundance.

For all chemical elements presented in Fig. 7, the abundance difference between zones of the highest and lowest concentrations does not exceed $1 \mathrm{dex}$. For $\mathrm{Ba}$ the range is $0.81 \mathrm{dex}$, for $\mathrm{Y}$ it is 0.71 dex. For $\mathrm{Ti}$ and $\mathrm{Sr}$ the range is $0.36 \mathrm{dex}$ and 0.59 dex, respectively. This agrees well with the relative amplitude of variability in individual line profiles of these chemical elements (Fig. 6).
To exclude the possibility that spots are found by the DI code at roughly one hemisphere of the star owing to a spurious systematic effect, we applied the same DI analysis to ten spectral lines of $\mathrm{Fe}$, which show no variability. The inferred abundance map of $\mathrm{Fe}$ is not similar to the maps of other chemical elements in its morphology. In particular, it does not show an abundance difference between the two hemispheres. The difference between the regions of the highest and lowest abundances does not exceed $\approx 0.15 \mathrm{dex}$, which we take to be an upper limit of the possible inhomogeneity for chemical elements without obvious line profile variability.

\section{Summary and discussion}

Despite singular magnetic field detections occasionally claimed for HgMn stars (e.g. Hubrig et al. 2010), all systematic attempts to find the magnetic fields that could be responsible for chemical inhomogeneities were unsuccessful (Shorlin et al. 2002; Wade et al. 2006; Folsom et al. 2010; Aurière et al. 2010; Makaganiuk et al. 2011).

To extend the group of spotted HgMn stars with known constraints on their magnetic field, we performed time-series spectropolarimetric observations of 66 Eri. This is the first such analysis of this unique spectroscopic binary star. It allowed us to search for magnetic fields in both components and investigate their line profile variability.

Taking the advantage of one of the best spectropolarimeters in the world, we recorded high $S / N$ and high-resolution spectra of 66 Eri covering its full orbital period. Despite the high polarimetric sensitivity of the instrument, we did not find any evidence of the longitudinal magnetic field in either component of 66 Eri. Our measurements of $\left\langle B_{z}\right\rangle$ using LSD profiles have error bars 10-24 G for both stars. This null result agrees with the outcome of our earlier work (Makaganiuk et al. 2011), where the longitudinal magnetic field was measured in a sample of $47 \mathrm{HgMn}$ 
Table 5. List of variable lines in the primary component of 66 Eri.

\begin{tabular}{|c|c|c|c|}
\hline Ion & $\lambda(\AA)$ & Ion & $\lambda(\AA)$ \\
\hline $\mathrm{Hg}_{\text {II }}$ & 3983.931 & & \\
\hline Ti II & 4028.338 & Si II & 4028.465 \\
\hline Ti II & 4053.821 & Cr II & 4054.076 \\
\hline Sr II & 4077.709 & & \\
\hline $\mathrm{Y}_{\mathrm{II}}$ & 4124.907 & $\mathrm{Fe}_{\text {II }}$ & 4124.787 \\
\hline $\mathrm{Cr}$ II & 4275.567 & & \\
\hline Ti II & 4163.644 & & \\
\hline Ti II & 4171.904 & & \\
\hline Y II & 4177.529 & $\mathrm{Fe}_{\text {II }}$ & 4177.692 \\
\hline Y II & 4199.277 & $\mathrm{Fe}_{\mathrm{I}}$ & 4199.095 \\
\hline $\mathrm{Y}_{\mathrm{II}}$ & 4204.695 & & \\
\hline Sr II & 4215.519 & & \\
\hline Y II & 4235.729 & $\mathrm{Fe}_{\mathrm{I}}$ & 4235.937 \\
\hline Ti II & 4290.215 & & \\
\hline Y II & 4309.631 & & \\
\hline$Y_{\text {II }}$ & 4358.728 & & \\
\hline Ti II & 4367.652 & & \\
\hline$Y_{\text {II }}$ & 4374.935 & Ti II & 4374.816 \\
\hline Ti II & 4386.846 & & \\
\hline $\mathrm{Y}_{\mathrm{II}}$ & 4398.013 & Ti II & 4398.292 \\
\hline Ti II & 4421.938 & & \\
\hline$Y_{\text {II }}$ & 4422.591 & & \\
\hline $\mathrm{Ba}_{\text {II }}$ & 4554.029 & & \\
\hline Ti II & 4563.757 & & \\
\hline Ti II & 4571.971 & & \\
\hline Ti II & 4589.947 & $\mathrm{Cr}_{\text {II }}$ & 4589.901 \\
\hline $\mathrm{Cr}_{\text {II }}$ & 4592.049 & & \\
\hline $\mathrm{Cr}_{\text {II }}$ & 4634.070 & & \\
\hline $\mathrm{Y}_{\mathrm{II}}$ & 4682.324 & & \\
\hline Ti II & 4779.985 & & \\
\hline Ti II & 4805.085 & & \\
\hline Cr II & 4812.337 & & \\
\hline $\mathrm{Y}_{\mathrm{II}}$ & 4823.304 & & \\
\hline$Y_{\text {II }}$ & 4883.684 & & \\
\hline$Y_{\text {II }}$ & 4900.120 & Ba II & 4899.929 \\
\hline Ti II & 4911.195 & & \\
\hline Ba II & 4934.076 & & \\
\hline Y II & 4982.129 & & \\
\hline$Y_{\text {II }}$ & 5087.416 & & \\
\hline$Y_{\text {II }}$ & 5119.112 & & \\
\hline$Y_{\text {II }}$ & 5123.211 & & \\
\hline Ti II & 5129.156 & & \\
\hline Ti II & 5185.902 & & \\
\hline Ti II & 5188.687 & & \\
\hline Y II & 5196.422 & & \\
\hline$Y_{\text {II }}$ & 5200.406 & & \\
\hline$Y_{\text {II }}$ & 5205.724 & & \\
\hline$Y_{\text {II }}$ & 5473.388 & & \\
\hline$Y_{\text {II }}$ & 5480.732 & & \\
\hline$Y_{\text {II }}$ & 5497.408 & & \\
\hline $\mathrm{Y}_{\text {II }}$ & 5509.895 & & \\
\hline$Y_{\text {II }}$ & 5521.555 & & \\
\hline $\mathrm{Y}_{\mathrm{II}}$ & 5544.611 & & \\
\hline Y II & 5546.009 & & \\
\hline $\mathrm{Y}_{\text {II }}$ & 5662.925 & & \\
\hline Y II & 6795.414 & & \\
\hline
\end{tabular}

Notes. Bold font marks the spectral lines used for Doppler imaging and italics indicate lines with marginal variability.

stars. The analysis of these objects showed no evidence of weak magnetic field signatures in Stokes $V$ spectra.

Our magnetic field measurements provide an upper limit of 60-70 $\mathrm{G}$ for a dipolar field in either star and LSD $V$ profiles provide no evidence for the presence of strong, complex magnetic field structures. This upper limit is significantly lower than the minimum dipolar field diagnosed in Ap stars (Aurière et al. 2007). Thus, 66 Eri is clearly not an Ap star.

Because we deal with the binary system that shows two systems of spectral lines in its composite spectrum, it is necessary to disentangle the spectra to study each component separately. This was accomplished with the procedure of spectral disentangling. Along with the intrinsic spectra of the components it provided their radial velocities. The fit of our RV measurements together with RVs from previous studies (Young 1976; Yushchenko et al. 1999, 2001; Catanzaro \& Leto 2004) with the theoretical model enabled us to improve the orbital elements of 66 Eri.

Using constraints from the orbital solution, we found the fundamental parameters for each star with the help of evolutionary tracks. The primary $(\mathrm{HgMn})$ star turned out to be more luminous and hotter, as expected. Yushchenko et al. (1999) considered the $\mathrm{HgMn}$ star to be the secondary component in their abundance analysis. The effective temperature revision of $200 \mathrm{~K}$ suggested in our study is relatively unimportant for abundance determination.

The evolutionary tracks provided masses of the components, which were utilized to compute the orbital inclination angle. The other method, based on the oblique rotator model, yielded nearly the same value for the rotational inclination angles. These results indicate that the angle between the rotation axes of two stars and the orbital plane is $90^{\circ}$ and their rotation is synchronized with the orbital motion.

For the identification of spectral lines, we computed a synthetic spectrum based on the abundances provided by Yushchenko et al. (1999) for both components. For the primary component the agreement between our synthetic spectrum and observations is very good in most cases, in particular for the lines of $\mathrm{Fe}, \mathrm{Ti}, \mathrm{Cr}, \mathrm{Y}, \mathrm{Ba}, \mathrm{Si}, \mathrm{Mn}$, and $\mathrm{Hg}$. At the same time, we cannot confirm the high abundances of $\mathrm{La}, \mathrm{Zr}, \mathrm{Yb}, \mathrm{Hf}, \mathrm{Pt}$, $\mathrm{Au}$, and $\mathrm{W}$ inferred by Yushchenko et al. (1999). The strongest lines of these elements are very weak or absent in the observations, while in the synthetic spectrum they are too strong. The abundances of all these elements, derived by Yushchenko et al. (1999) based on a small number of very weak spectral lines, are not reliable. The abundances for the secondary component seem to agree very well with our observations.

In conclusion of their abundance analysis Yushchenko et al. (1999) made a confusing suggestion that the more massive component of 66 Eri is not an HgMn star. This claim contradicts the standard definition of an HgMn star as a late-B, slowly rotating object with enhanced $\mathrm{Hg}$ or Mn lines (e.g. Preston 1974). Yushchenko et al. (1999) themselves found both $\mathrm{Hg}$ and $\mathrm{Mn}$ to be overabundant; a high $\mathrm{Hg}$ overabundance was confirmed by Woolf \& Lambert (1999). The absence of P overabundance is unusual, but is known for several other $\mathrm{HgMn}$ stars with $T_{\text {eff }}$ similar to 66 Eri A (Adelman et al. 2001), while a significant Ga overabundance is mostly inferred from the optical spectra of $\mathrm{HgMn}$ stars much hotter than 66 Eri A (Ryabchikova et al. 1996). Thus, 66 Eri A is undoubtedly an HgMn star and was treated as such in many modern studies (Cowley et al. 2007; Catanzaro \& Leto 2004; Rottler \& Young 2002; Woolf \& Lambert 1999; Hubrig 1998). 66 Eri is also classified as " $\mathrm{Hg}$ " in the updated "Catalogue of Ap, HgMn and Am stars" (Renson \& Manfroid 2009).

The analysis of spectral lines in the disentangled spectra of 66 Eri A allowed us to discover the variability in some of them. This was not achieved in the previous studies of this star because of a low resolving power and poor $S / N$. On the other hand, the same analysis revealed no variable spectral lines for 66 Eri B.

The spectroscopic variability of 66 Eri A turned out to be different from other $\mathrm{HgMn}$ stars studied previously with time-series 
V. Makaganiuk et al.: Magnetic fields and variability of 66 Eri

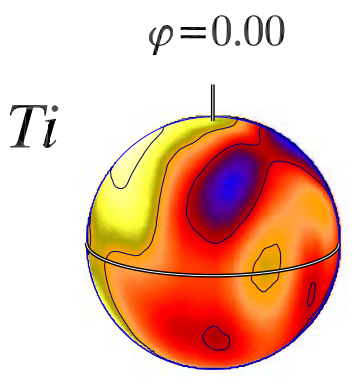

$$
\varphi=0.20
$$

$\varphi=0.40$

$\varphi=0.60$
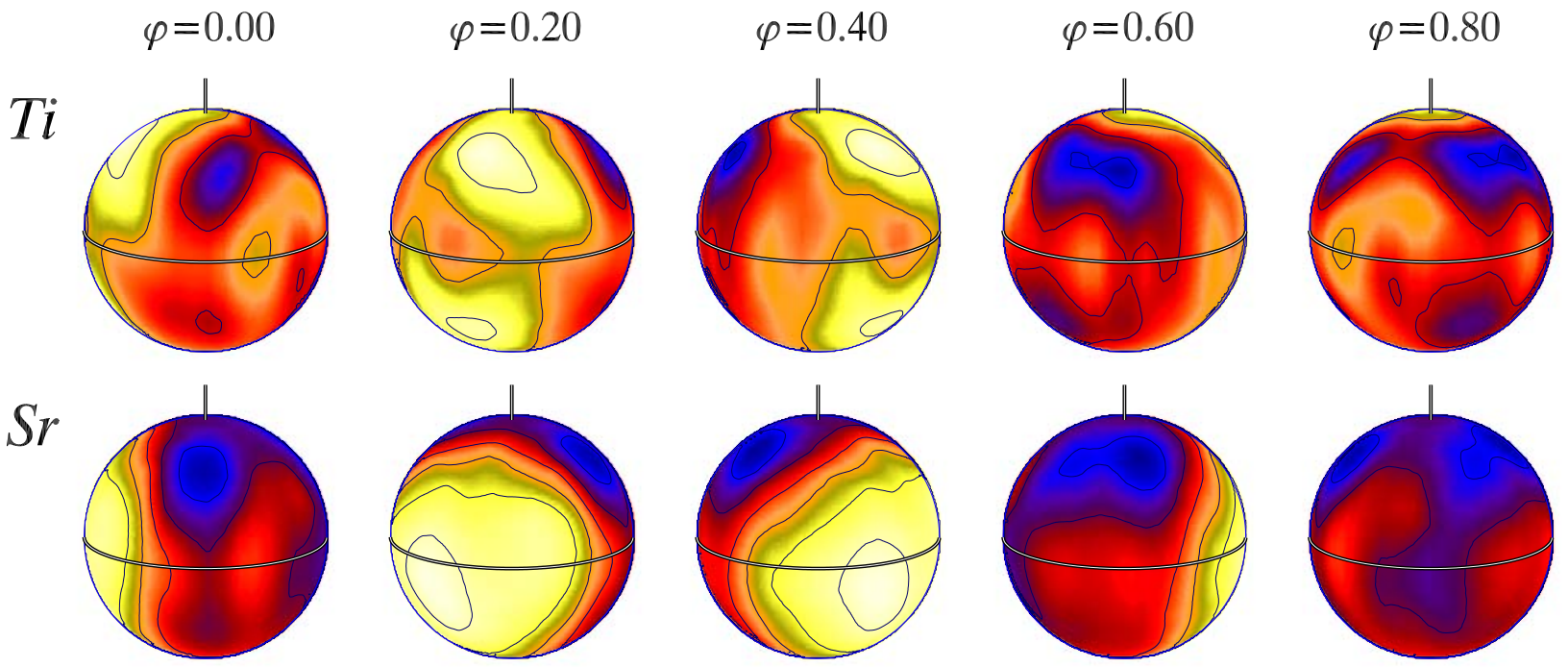

$\overline{\mathrm{Sr}]}$

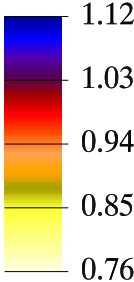
1.45
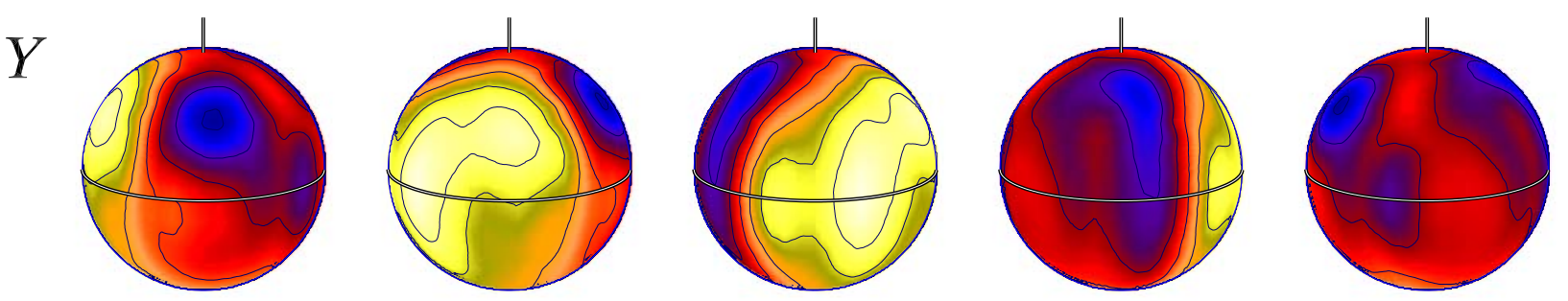

[Y]
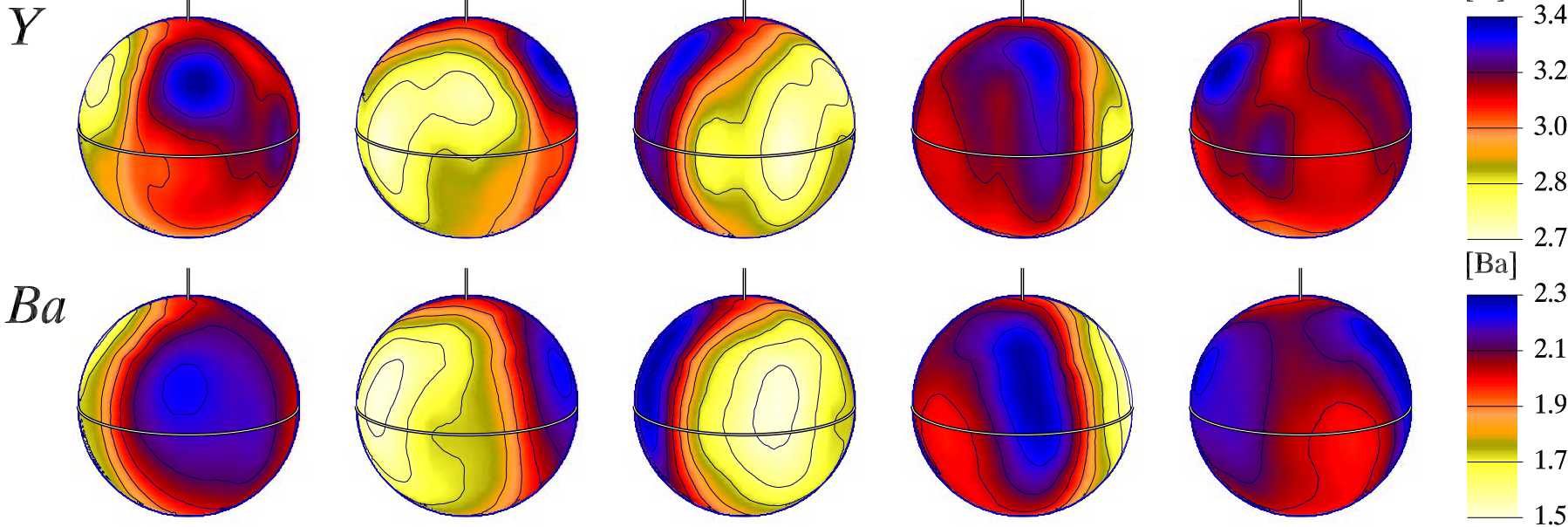

[Ba]

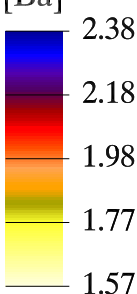

Fig. 7. Surface distributions of Ti, Sr, Y, and Ba abundances reconstructed for 66 Eri A. The star is shown at five equidistant rotation phases at the inclination angle $i=75^{\circ}$ and vertically oriented axis of rotation. The darker spots represent a higher concentration of chemical elements. The contours of equal abundance are plotted with a step of 0.1 dex for all chemical species. The thick line indicates the position of rotational equator, while the vertical bar shows rotational pole. The scale of the abundance maps is shown on the right. Abundances are given in a logarithmic scale relative to the Sun.

spectroscopy. All of them show notable variability in the $\mathrm{Hg}$ II spectral lines. For example, for $\alpha$ And Kochukhov et al. (2007) reported $\approx 30 \%$ equivalent width changes of the $\mathrm{Hg}$ II $3984 \AA$ line. The variability in this line is barely detectable in 66 Eri A. Our spectra are also insufficient to unambiguously show variability in $\mathrm{Cr}$ lines, although we found marginal changes in four of them.

More pronounced variability is found for the spectral lines of Y, Sr, Ba, and Ti. In Fig. 6 we illustrate the line profiles of these four chemical elements that possess the strongest variability among all spectral lines of each species. The change in profiles with the rotational phase is still rather weak compared to other spotted HgMn stars, which makes this star the least variable.

We found a slightly different line profile variability pattern for four elements. This indicates a difference in the distribution of these chemical elements over the surface of 66 Eri A. We applied the Doppler imaging technique to study the spot geometry. The derived surface maps (Fig. 7) indicate higher concentrations of chemical elements on one hemisphere of the star. The overall abundance contrast is about 0.5 dex for all studied chemical elements. The hemisphere with a lower abundance still has higher concentrations of these elements compared to the Sun.
This means that a certain physical process leads to a systematic difference in abundances between the two hemispheres. This phenomenon cannot be attributed to the strong magnetic field because it is absent from the atmosphere of 66 Eri A.

Low-contrast abundance inhomogeneities found on the surface of 66 Eri A may give rise to photometric variability. So far the evidence of light variation in 66 Eri was controversial. Yushchenko et al. (1999) could not verify the weak photometric variability suggested by (Schneider 1987), but suggested the presence of variability with half of the orbital period in the Hipparcos epoch photometry of 66 Eri. Our reanalysis of these data does not support the claim by Yushchenko et al. There is a peak in the amplitude spectrum at $P=2.754 \pm 0.004 \mathrm{~d}$, which is close to, but formally is not consistent with, $P_{\text {orb }} / 2=$ $2.761 \mathrm{~d}$. However, this periodicity is not statistically significant $(\mathrm{FAP}=0.4)$ and there are more than ten other peaks in the amplitude spectrum with the same or higher magnitude.

Because 66 Eri is a binary system, we cannot exclude the influence of the secondary component on the atmosphere of the primary. To understand how the position of chemical spots in 66 Eri A correlates with the relative orbital motion of the components, we combined the results of the orbital analysis and DI maps of yttrium. The resulting picture is shown in Fig. 11. 


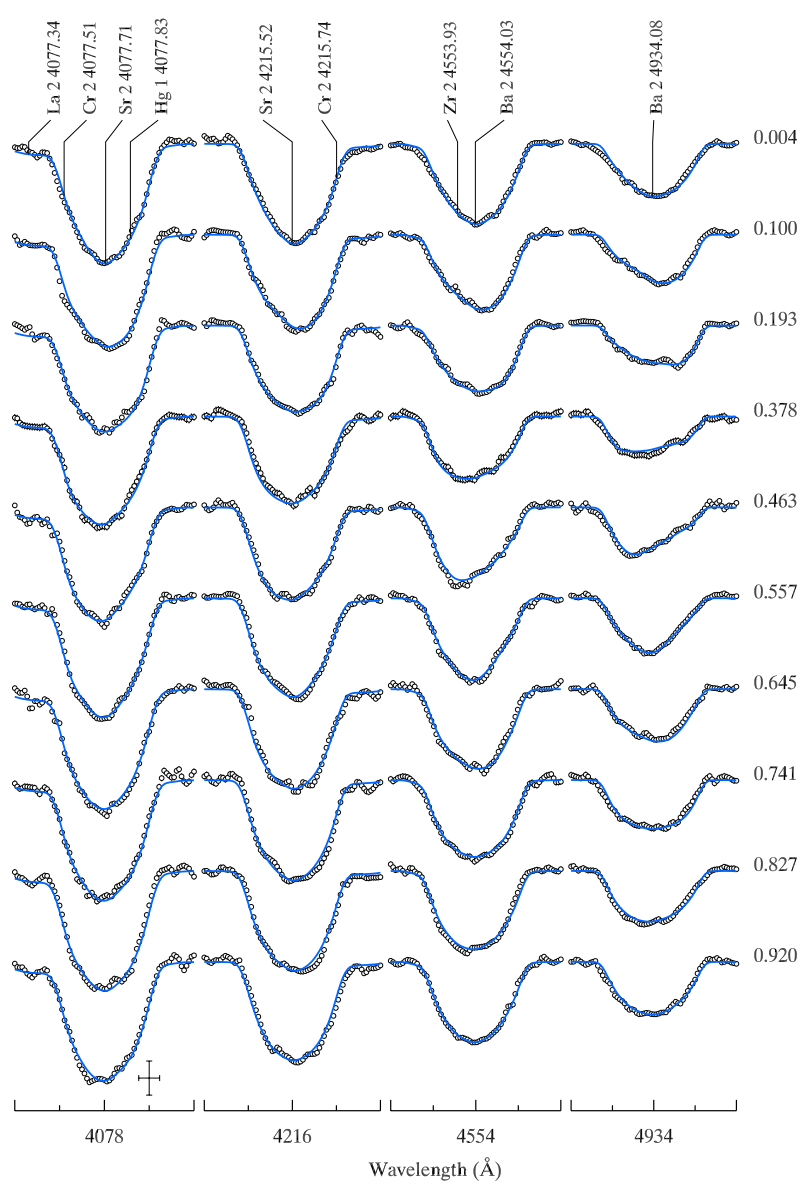

Fig. 8. Comparison of the observed (symbols) and model (solid lines) profiles for Sr and Ba lines in 66 Eri A. The bars in the lower left corner indicate the horizontal and vertical scales $(0.1 \AA$ and $5 \%$ of the continuum intensity respectively).

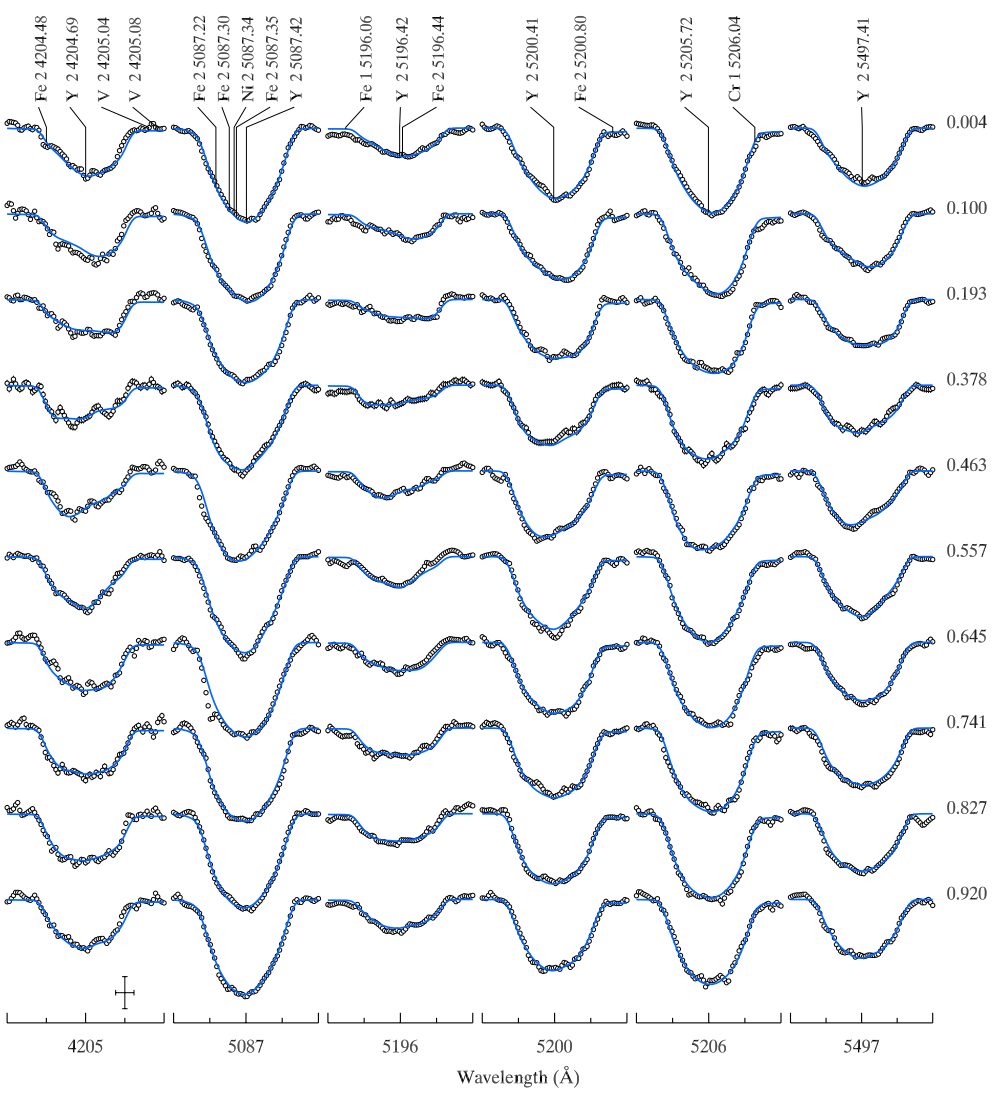

Fig. 9. Same as Fig. 8 but for Y. 
V. Makaganiuk et al.: Magnetic fields and variability of 66 Eri

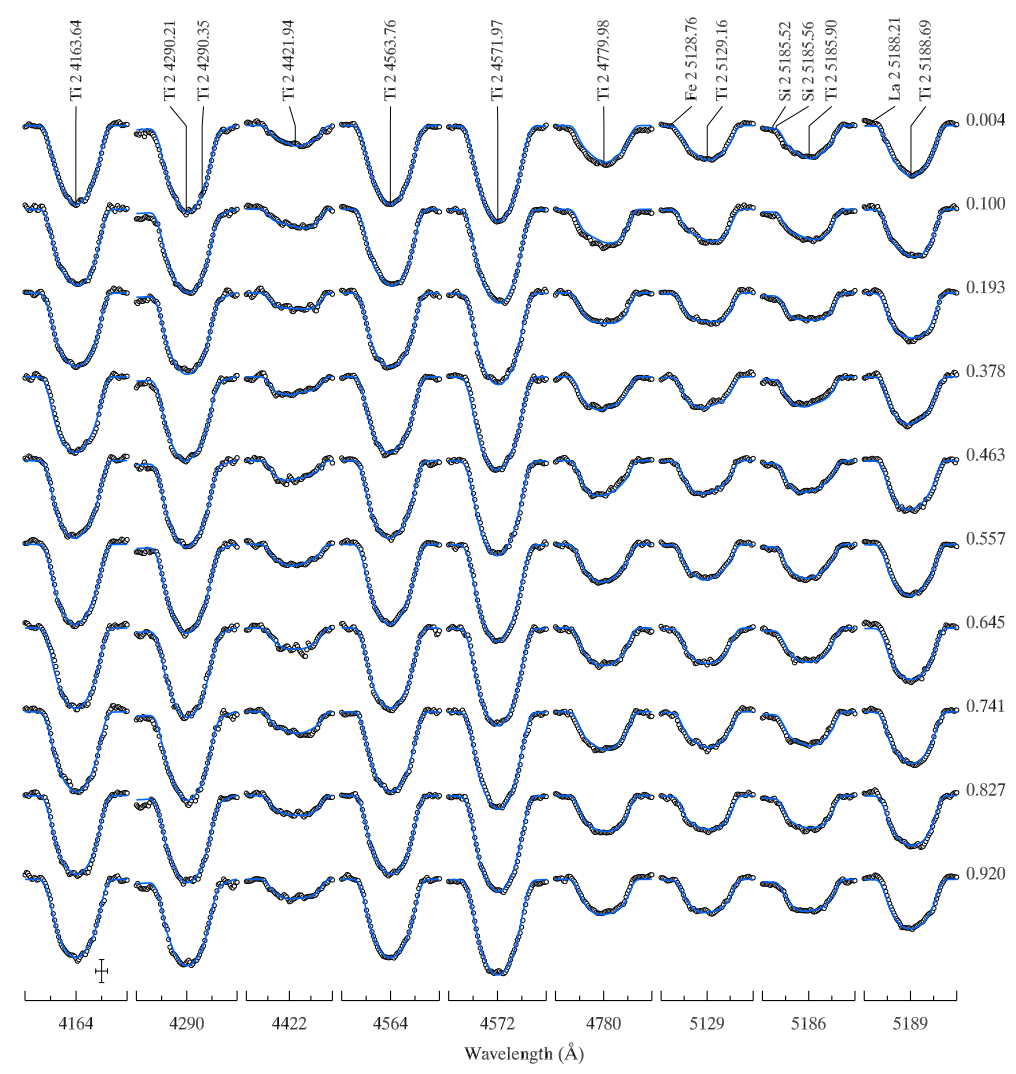

Fig. 10. Same as Fig. 8 but for Ti.
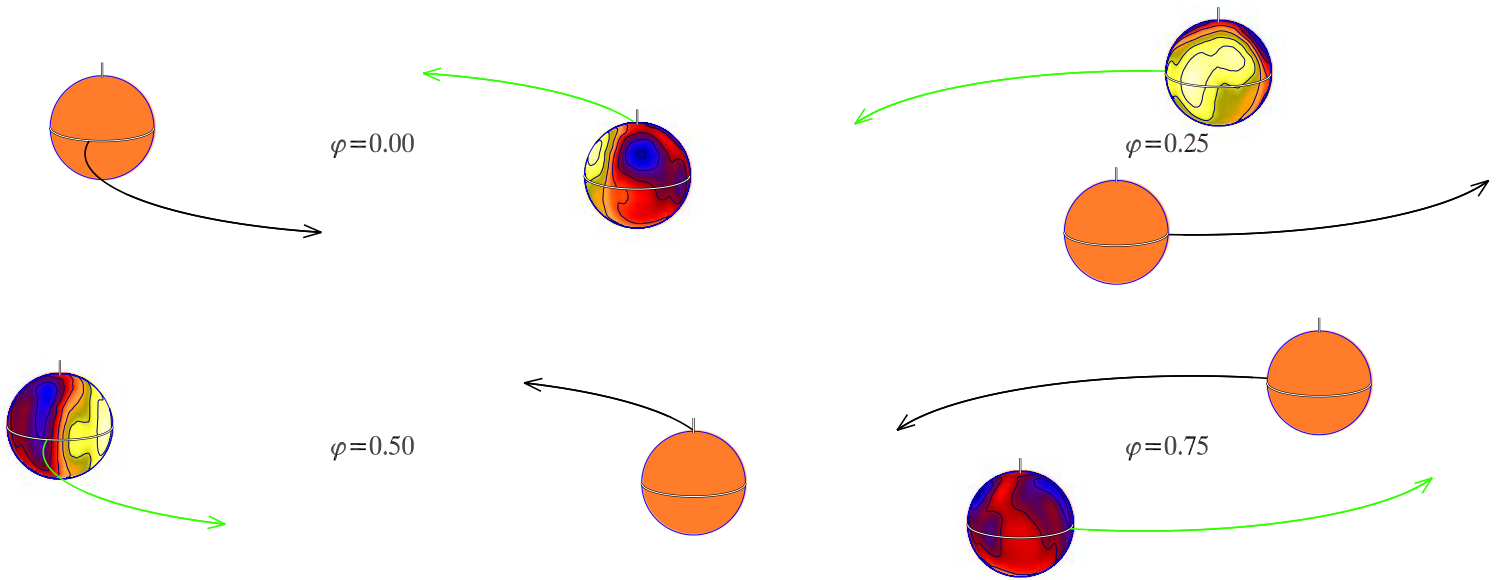

Fig. 11. Orbital motion and rotation of the components in the binary system 66 Eri. The star with yttrium spots represents 66 Eri A and the other filled with a solid colour represents 66 Eri B. The radii and the separation between the two stars are shown to scale.

It turns out that the relative underabundance of $\mathrm{Ti}, \mathrm{Sr}, \mathrm{Y}$, and $\mathrm{Ba}$ in 66 Eri $\mathrm{A}$ is observed on the hemisphere that faces 66 Eri B. Owing to a fair proximity of the two stars, the tidal effects can be responsible for the destruction of the upper layer that contains the excess of the studied elements. On the other hand, the radiation flux from 66 Eri B can also have an impact on the distribution of the chemical elements in the atmosphere of the primary component. However, it is not clear why only one of the nearly identical stars is affected by these effects.

A relative element underabundance on the side facing the secondary was reported for Y in AR Aur A (Hubrig et al. 2010), although their maps, reconstructed from a single blended Y II line, are dominated by the equator-to-pole variation. An earlier Y map by Savanov et al. (2009) showed a different abundance spot topology. The interpretation of the AR Aur maps is difficult because of the well-known problem in the Doppler imaging of equator-on stars. This intrinsic ambiguity can be resolved by incorporating observations during eclipse phases in the abundance inversion (Vincent et al. 1993).

In addition to the puzzling spectroscopic variability in 66 Eri A, the two stars have significantly different average chemical characteristics. Despite the two stars having nearly the same fundamental characteristics, the secondary component seems to be a normal star with nearly solar chemical composition and shows no surface inhomogeneities. Remarkably, the line profile variability in the A component occurs only for those elements that show much stronger spectral lines in the primary. This is also clearly seen from the abundances of $\mathrm{Ti}, \mathrm{Sr}, \mathrm{Y}$, and $\mathrm{Ba}$ 
determined by Yushchenko et al. (1999): all these four elements are significantly more abundant in the atmosphere of the primary. Among the elements with the largest abundance difference between the components only $\mathrm{Hg}$ does not clearly show a nonuniform distribution.

The identical age of both stars and the small separation between them suggest that they were formed in the same protostellar gas cloud. This would imply the same overall chemical composition for both stars. The observed chemical inhomogeneities in one of the two components and a large difference between their average abundance could be related. These effects cannot be attributed to the generic atomic diffusion, which only depends on fundamental stellar parameters. It must be a result of some rapid process that changes the atomic diffusion on a timescale much shorter than the age of a star. If this is true, the secondary component has either already undergone the stage of non-uniform surface distribution of chemical elements or may show surface structures in the future.

Acknowledgements. We thank the referee David Bohlender who helped us to make some points more clear. O.K. is a Royal Swedish Academy of Sciences Research Fellow, supported by grants from Knut and Alice Wallenberg Foundation and Swedish Research Council.

\section{References}

Adelman, S. J., Gulliver, A. F., \& Rayle, K. E. 2001, A\&A, 367, 597

Adelman, S. J., Gulliver, A. F., Kochukhov, O. P., \& Ryabchikova, T. A. 2002, ApJ, 575, 449

Aurière, M., Wade, G. A., Silvester, J., et al. 2007, A\&A, 475, 1053

Aurière, M., Wade, G. A., Konstantinova-Antova, R., et al. 2009, A\&A, 504, 231

Aurière, M., Wade, G. A., Lignières, F., et al. 2010, A\&A, 523, A40

Bagnulo, S., Landolfi, M., Landstreet, J. D., et al. 2009, PASP, 121, 993

Berghofer, T. W., \& Schmitt, J. H. M. M. 1994, A\&A, 292, L5

Briquet, M., Korhonen, H., González, J. F., Hubrig, S., \& Hackman, T. 2010, A\&A, 511, A71

Catanzaro, G., \& Leto, P. 2004, A\&A, 416, 661

Cowley, C. R., Hubrig, S., Castelli, F., González, J. F., \& Wolff, B. 2007, MNRAS, 377, 1579

Donati, J., \& Landstreet, J. D. 2009, ARA\&A, 47, 333

Donati, J., Semel, M., Carter, B. D., Rees, D. E., \& Collier Cameron, A. 1997, MNRAS, 291, 658
Folsom, C. P., Kochukhov, O., Wade, G. A., Silvester, J., \& Bagnulo, S. 2010, MNRAS, 407, 2383

Frost, E. B., \& Struve, O. 1924, ApJ, 60, 313

Girardi, L., Bressan, A., Bertelli, G., \& Chiosi, C. 2000, A\&AS, 141, 371

Hubrig, S. 1998, Contributions of the Astronomical Observatory Skalnate Pleso, 27, 296

Hubrig, S., Le Mignant, D., North, P., \& Krautter, J. 2001, A\&A, 372, 152

Hubrig, S., González, J. F., Savanov, I., et al. 2006, MNRAS, 371, 1953

Hubrig, S., Savanov, I., Ilyin, I., et al. 2010, MNRAS, 408, L61

Khokhlova, V. L., Zverko, Y., Zhizhnovskii, I., \& Griffin, R. E. M. 1995, Astr. Lett., 21, 818

Kochukhov, O., Piskunov, N., Sachkov, M., \& Kudryavtsev, D. 2005, A\&A, 439, 1093

Kochukhov, O., Adelman, S. J., Gulliver, A. F., \& Piskunov, N. 2007, Nature Physics, 3, 526

Kochukhov, O., Makaganiuk, V., \& Piskunov, N. 2010, A\&A, 524, A5

Leroy, J. L., Bagnulo, S., Landolfi, M., \& Landi Degl'Innocenti, E. 1994, A\&A, 284,174

Lignières, F., Petit, P., Böhm, T., \& Aurière, M. 2009, A\&A, 500, L41

Makaganiuk, V., Kochukhov, O., Piskunov, N., et al. 2011, A\&A, 525, A97

Mayor, M., Pepe, F., Queloz, D., et al. 2003, The Messenger, 114, 20

Piskunov, N., \& Kochukhov, O. 2002a, A\&A, 381, 736

Piskunov, N. E., \& Valenti, J. A. 2002b, A\&A, 385, 1095

Piskunov, N. E., Kupka, F., Ryabchikova, T. A., Weiss, W. W., \& Jeffery, C. S. 1995, A\&AS, 112, 525

Preston, G. W. 1974, ARA\&A, 12, 257

Renson, P., \& Manfroid, J. 2009, A\&A, 498, 961

Rottler, L., \& Young, A. 2002, in BAAS, 201, 404

Ryabchikova, T. A., Zakharova, L. A., \& Adelman, S. J. 1996, MNRAS, 283, 1115

Savanov, I. S., Hubrig, S., González, J. F., \& Schöller, M. 2009, in IAU Symp., 259,401

Schneider, H. 1987, Hvar Observatory Bulletin, 11, 29

Shorlin, S. L. S., Wade, G. A., Donati, J., et al. 2002, A\&A, 392, 637

Snik, F., Jeffers, S., Keller, C., et al. 2008, in SPIE Conf. Ser., 7014, 22

Snik, F., Kochukhov, O., Piskunov, N., et al. 2010, ASP Conf. Ser., in press [arXiv: 1010.0397]

Stelzer, B., Huélamo, N., Micela, G., \& Hubrig, S. 2006, A\&A, 452, 1001

van Leeuwen, F., \& Fantino, E. 2005, A\&A, 439, 791

Vincent, A., Piskunov, N. E., \& Tuominen, I. 1993, A\&A, 278, 523

Wade, G. A., Donati, J., Landstreet, J. D., \& Shorlin, S. L. S. 2000, MNRAS, 313,823

Wade, G. A., Aurière, M., Bagnulo, S., et al. 2006, A\&A, 451, 293

Woolf, V. M., \& Lambert, D. L. 1999, ApJ, 521, 414

Young, A. 1976, PASP, 88, 275

Yushchenko, A. V., Gopka, V. F., Khokhlova, V. L., Musaev, F. A., \& Bikmaev, I. F. 1999, Astr. Lett., 25, 453

Yushchenko, A. V., Gopka, V. F., Khokhlova, V. L., \& Tomkin, J. 2001, Information Bulletin on Variable Stars, 5213, 1 